\title{
Functional impact of global rare copy number variation in autism spectrum disorders
}

\author{
A list of authors and their affiliations appears at the end of the paper.
}

The autism spectrum disorders (ASDs) are a group of conditions characterized by impairments in reciprocal social interaction and communication, and the presence of restricted and repetitive behaviours ${ }^{1}$. Individuals with an ASD vary greatly in cognitive development, which can range from above average to intellectual disability $^{2}$. Although ASDs are known to be highly heritable $(\sim 90 \%)^{3}$, the underlying genetic determinants are still largely unknown. Here we analysed the genome-wide characteristics of rare ( $<1 \%$ frequency) copy number variation in ASD using dense genotyping arrays. When comparing 996 ASD individuals of European ancestry to 1,287 matched controls, cases were found to carry a higher global burden of rare, genic copy number variants (CNVs) (1.19 fold, $P=0.012$ ), especially so for loci previously implicated in either ASD and/or intellectual disability (1.69 fold, $\left.P=3.4 \times 10^{-4}\right)$. Among the CNVs there were numerous de novo and inherited events, sometimes in combination in a given family, implicating many novel ASD genes such as SHANK2, SYNGAP1, DLGAP2 and the X-linked DDX53-PTCHD1 locus. We also discovered an enrichment of CNVs disrupting functional gene sets involved in cellular proliferation, projection and motility, and GTPase/Ras signalling. Our results reveal many new genetic and functional targets in ASD that may lead to final connected pathways.

Twin and family studies indicate a predominantly genetic basis for ASD susceptibility and provide support for considering these disorders as a clinical spectrum. Some $5-15 \%$ of individuals with an ASD have an identifiable genetic aetiology corresponding to known rare single-gene disorders (for example, fragile $\mathrm{X}$ syndrome) and chromosomal rearrangements (for example, maternal duplication of 15q11-q13). Rare mutations have been identified in synaptic genes, including NLGN3, NLGN4X (ref. 4) and SHANK3 (ref. 5), and microarray studies have revealed copy number variation $(\mathrm{CNV})$ as risk factors ${ }^{6}$. CNV examples include de novo events observed in $5-10 \%$ of ASD cases $^{7-9}$, de novo or inherited hemizygous deletions and duplications of 16p11.2 (refs 9-11) and NRXN1 (ref. 7), and exceptionally rare homozygous deletions in consanguineous families ${ }^{12}$. Genomewide association studies using single nucleotide polymorphisms (SNPs) have highlighted two potential ASD risk loci at 5p14.1 (ref. 13) and 5p15.2 (ref. 14), but these data indicate that common variation will account for only a small proportion of the heritability in ASD.

To delineate further the contribution of rare genomic variants to autism we genotyped 1,275 ASD cases and their parents using the Illumina Infinium 1M single SNP microarray (Fig. 1). A set of 1,981 controls used for comparison studies was genotyped on the same platform ${ }^{15}$ and both data sets were subjected to the same quality control procedures. Ultimately, we analysed 996 ASD cases ( 876 trios) and 1,287 controls of European ancestry to minimize confounds due to population differences (Supplementary Figs 1 and 2 and Supplementary Table 1$)^{16}$.

Two CNV prediction algorithms (QuantiSNP ${ }^{17}$ and iPattern (unpublished data)) and additional extensive quality control procedures were used to establish a stringent data set of non-redundant CNVs called by both algorithms in an individual (Fig. 1, Supplementary Tables 1-3 and Supplementary Fig. 3). This stringent data set of 5,478 rare CNVs in 996 cases and 1,287 controls of European ancestry (Supplementary Table 4) had the following characteristics: (1) CNV present at $<1 \%$ frequency in the total sample (cases and controls); (2) $\mathrm{CNV} \geq 30 \mathrm{~kb}$ in size (because $>95 \%$ of these could be confirmed); and (3) all CNVs further verified using combined evidence from the PennCNV algorithm ${ }^{18}$ and child-parent intensity fold changes, genotype proportions (to verify deletions) and visual inspection (for chromosome $\mathrm{X}$ ).

We assessed the impact of rare CNV in cases compared to controls using three primary measures of CNV burden: the number of CNVs per individual, the estimated CNV size, and the number of genes affected by CNVs (Table 1). No significant difference was found in the former two

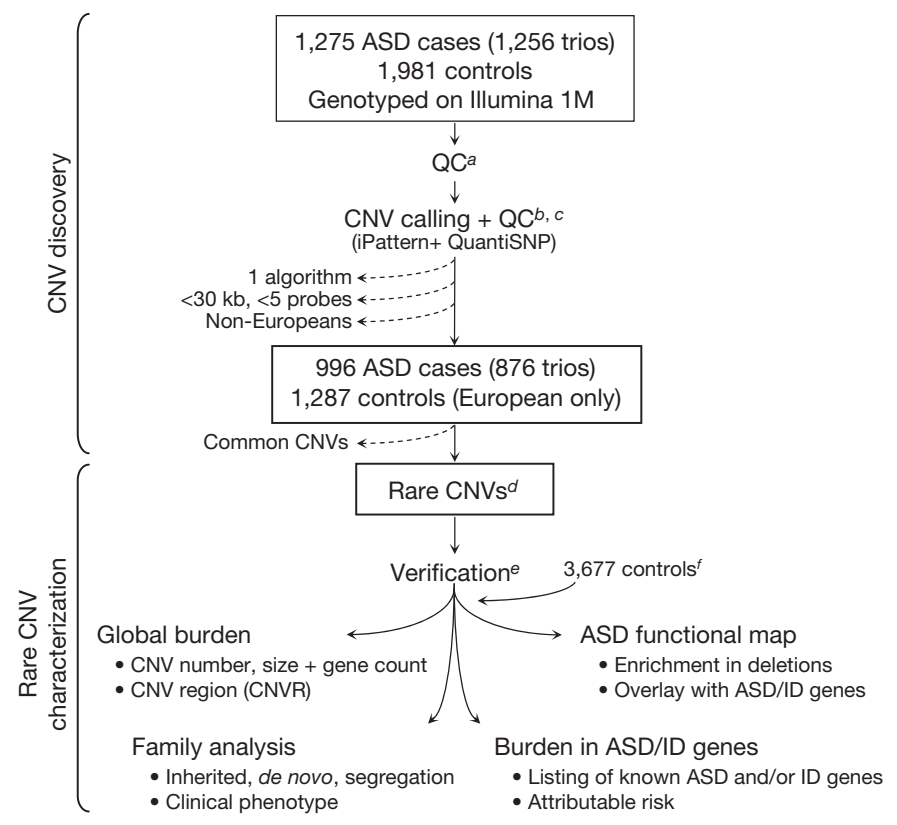

Figure 1 | CNV discovery and characterization. Comprehensive procedures were used to identify the rare CNV data set (boxed). Dashed arrows indicate CNVs not included in downstream analyses. Labels $a-f$ are as follows: $a$, SNP and intensity quality control (QC) with ancestry estimation; $b$, QC for CNV calls; $c$, pilot validation experiments using quantitative PCR were used to evaluate the false discovery rate; $d$, rare CNVs in samples of European ancestry were defined as $\geq 30 \mathrm{~kb}$ in size and present in the total sample set at a frequency $<1 \%$. A total of 70 out of $996(17 \%)$ of ASD cases were analysed on different lower-resolution arrays in previous studies ${ }^{9,10,28}$. Label $e$ indicates that all CNVs were computationally verified and at least $40 \%$ of case CNVs were also experimentally validated by qPCR and/or independent Agilent or other SNP microarrays; $f, 3,677$ additional European ancestry controls were used to test specific loci from the primary burden analyses. Additional details are in the Methods and Supplementary Information. ID, intellectual disability. 
Table 1 | Global burden of genic rare CNVs in cases versus controls

\begin{tabular}{|c|c|c|c|c|c|c|}
\hline Type & Classification & $\begin{array}{c}\text { Total } \\
\text { CNVs }(n)\end{array}$ & $P$ & Case/control ratio & Baseline rate (ctrl) & $P_{\text {corr }}$ \\
\hline & None & & & & & \\
\hline All & All & 5,478 & $0.012 *$ & 1.19 & 3.59 & $0.003 *$ \\
\hline Deletions only & All & 2,757 & $0.008 *$ & 1.26 & 1.08 & $0.006 *$ \\
\hline Duplications only & $\begin{array}{c}\text { All } \\
\text { CNV frequency }\end{array}$ & 2,721 & 0.072 & 1.16 & 2.51 & $0.015^{*}$ \\
\hline \multirow[t]{2}{*}{ All } & $2-6 x$ & 1,831 & 0.058 & 1.26 & 1.03 & 0.062 \\
\hline & $1 \times$ & 1,419 & 0.375 & 1.03 & 0.83 & 0.180 \\
\hline \multirow[t]{2}{*}{ Deletions only } & $2-6 x$ & 1,094 & $0.004^{*}$ & 1.57 & 0.43 & $0.011 *$ \\
\hline & $1 \times$ & 880 & $0.036 *$ & 1.26 & 0.30 & $0.036 *$ \\
\hline \multirow[t]{3}{*}{ Duplications only } & $2-6 x$ & 977 & 0.203 & 1.16 & 1.05 & 0.118 \\
\hline & $1 \times$ & 896 & 0.749 & 0.92 & 0.72 & 0.442 \\
\hline & CNV size & & & & & \\
\hline \multirow[t]{2}{*}{ All } & $30-500 \mathrm{~kb}$ & 5,086 & 0.313 & 1.03 & 2.72 & 0.181 \\
\hline & $\geq 500 \mathrm{~kb}$ & 392 & $0.005^{*}$ & 1.69 & 0.88 & $0.005 *$ \\
\hline \multirow[t]{2}{*}{ Deletions only } & $30-500 \mathrm{~kb}$ & 2,645 & $0.004 *$ & 1.24 & 0.85 & $0.004 *$ \\
\hline & $\geq 500 \mathrm{~kb}$ & 112 & 0.209 & 1.32 & 0.23 & 0.143 \\
\hline \multirow[t]{2}{*}{ Duplications only } & $30-500 \mathrm{~kb}$ & 2,441 & 0.801 & 0.93 & 1.86 & 0.518 \\
\hline & $\geq 500 \mathrm{~kb}$ & 280 & $0.007 *$ & 1.82 & 0.65 & $0.012 *$ \\
\hline
\end{tabular}

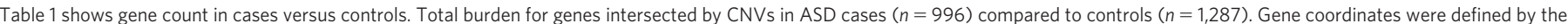

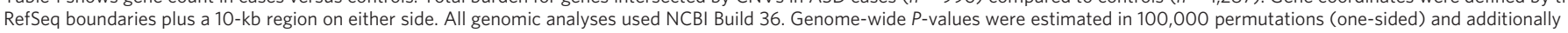

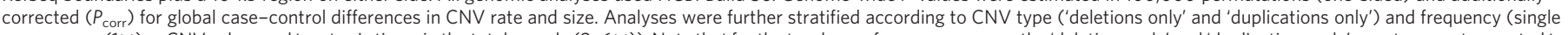

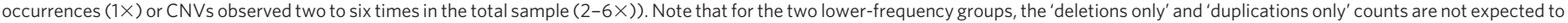
sum to the 'all' count (see ref. 26 and Supplementary Information).

* Significant differences $(P \leq 0.05)$ are indicated.

measures (Supplementary Tables 4a and 5), even after controlling for fine-level ancestry differences by pair-matching cases and controls (Supplementary Information $)^{16}$. In contrast, we discovered a significant increase in the number of genes intersected by rare CNV in cases when focusing on gene-containing segments (1.19-fold increase, empirical
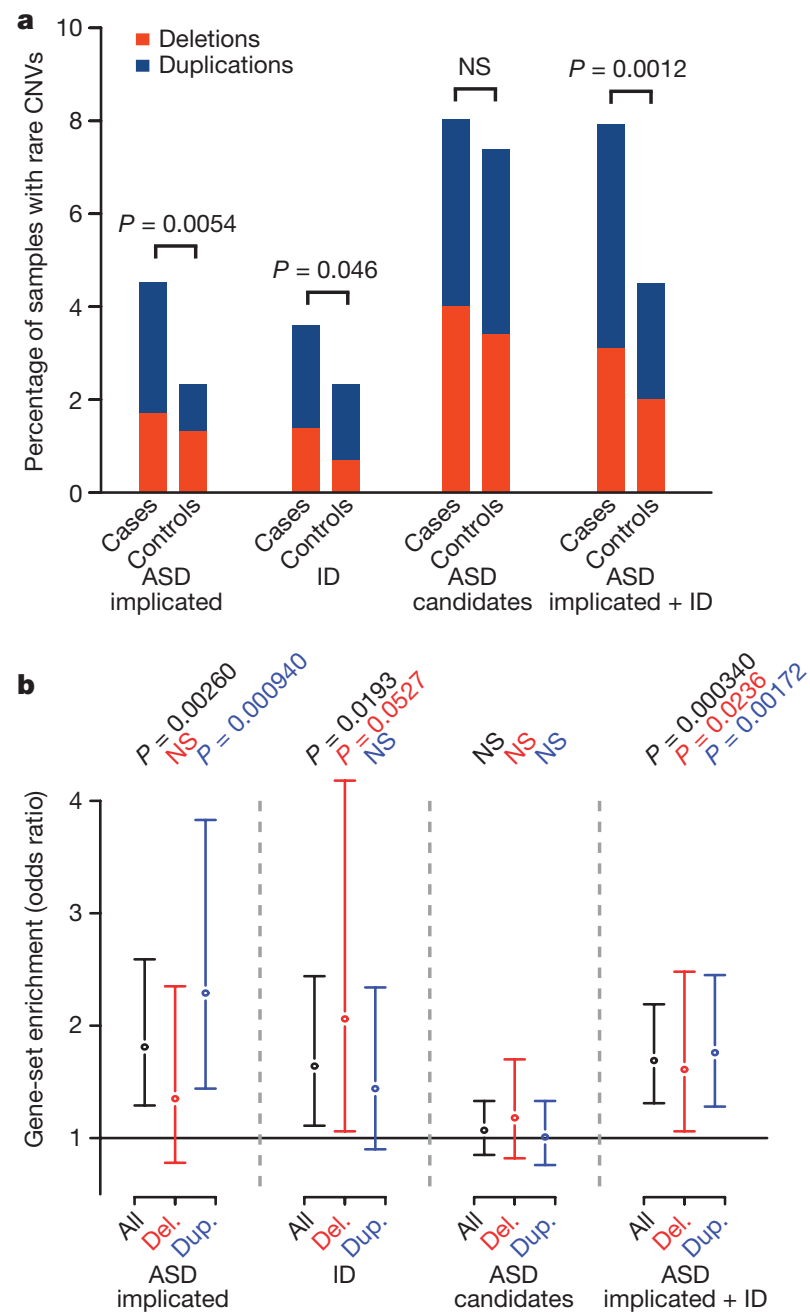

$P=0.012)$. This ASD association with genic CNV was stronger for deletions (1.26-fold increase, empirical $P=8.0 \times 10^{-3}$ ). These differences remained after we further controlled for potential case-control differences that could be present due to biological differences or technical biases. Restricting our analysis to autosomal CNVs (that is, after removing $\mathrm{CNV}$ s located on chromosome $\mathrm{X}$ ) also resulted in a consistent enriched gene count in ASD cases compared to controls. Singleoccurrence CNV deletions had increased rates in ASD cases over controls, indicating that some could be pathogenic.

We then examined parent-child transmission and confirmed that $5.7 \%$ (50 out of 876 ) of ASD cases had at least one de novo CNV with $>0.6 \%$ carrying two or more de novo events (Supplementary Tables $4 \mathrm{a}, 6$ and 7). The de novo $\mathrm{CNV}$ rate in our simplex and multiplex families was 5.6\% (22 out of 393) and 5.5\% (19 out of 348), respectively, in contrast with previous studies showing a higher rate in simplex families ${ }^{8,9}$. A total of 226 validated de novo (7) and inherited (219) CNVs not observed in controls and affecting single genes were found (Supplementary Table 8).

Numerous novel candidate ASD loci such as SHANK2, SYNGAP1 and DLGAP2 were identified on the basis of the observation that de novo CNV affects these genes in cases but not controls (Supplementary Table 6). The relatedness of SHANK2 to the causal ASD gene SHANK3 (ref. 5), involvement of SYNGAP1 in intellectual disability ${ }^{19}$, and interaction of DLGAP family proteins with SHANK proteins ${ }^{20}$ further support their role in ASDs. Maternally inherited X-linked deletions at DDX53-PTCHD1 (7 cases) implicate this locus in ASD. We tested an additional 3,677 European ancestry controls (Fig. 1) and again found no CNV at these genes, and DDX53-PTCHD1 emerged as a significant ASD risk factor $\left(P=3.1 \times 10^{-3}\right.$ with the initial 1,287 controls; $P=3.6 \times 10^{-6}$ with combined controls; Supplementary Fig. 4).

Figure 2 | CNV burden in known ASD and/or intellectual disability genes. a, Proportion of samples with CNVs overlapping genes and loci known to be associated in ASD with or without intellectual disability (ID) or intellectual disability only, as well as published candidate genes and loci for ASD (Supplementary Table 9). To select for CNVs with maximal impact, they needed to intersect genes and overlap the target loci by $\geq 50 \%$ of their length. Fisher's exact test $P$-values for significant differences ( $P \leq 0.05$, one tailed) are shown. NS, not significant. $\mathbf{b}$, Enrichment analysis for genes overlapped by rare CNVs in cases compared to controls for the three gene sets in a, relative to the whole genome. Odds ratio and $95 \%$ confidence intervals are given for each gene set. Empirical $P$-values for gene-set enrichment are indicated above each odds ratio. All $P$-values $<0.1$ are listed. 
Association studies of individual rare $\mathrm{CNV}$ often have insufficient power to discriminate benign from disease-causing variants. Here, we assessed whether genes and CNVs previously associated with ASD and/or intellectual disability were enriched in cases compared with controls, in order to help identify pathogenic events. We defined three gene lists based on evidence from previous studies of their involvement in ASDs (Supplementary Table 9): (1) 'ASD implicated' list consisting of 36 disease genes and 10 loci strongly implicated in ASD and identified in subjects with ASD or ASD and intellectual disability; (2) 'intellectual disability' consisting of 110 disease genes and 17 loci implicated in intellectual disability but not yet in ASD; and (3) 'ASD candidates' including 103 genes from previous studies of common and rare variants.

We observed a higher proportion of cases with rare CNVs overlapping 'ASD implicated' disease genes compared to controls (4.3\% versus $2.3 \%$, Fisher exact test $P=5.4 \times 10^{-3}$; Fig. 2a), corresponding to a significant enrichment for genes in this set (odds ratio $(\mathrm{OR})=1.8$; 95\% confidence interval (CI) $1.3-2.6$, empirical $P=2.6 \times 10^{-3}$; Fig. 2b, see also Supplementary Information). This effect was stronger for duplications, which may also disrupt genes $(\mathrm{OR}=2.3 ; 95 \% \mathrm{CI}$ 1.4-3.8, empirical $\left.P=9.4 \times 10^{-4}\right)$. Enrichment was also found for rare CNVs overlapping intellectual disability genes, more notably for deletions $(\mathrm{OR}=2.1 ; 95 \% \mathrm{CI} 1.1-4.2$, empirical $P=0.053)$. In contrast, there was no evidence of enrichment among case CNVs compared to control CNVs for genes in the ASD candidates set (empirical $P>0.3$ ). When the two disease gene sets 'ASD implicated' and 'intellectual disability' were combined, we observed $7.6 \%$ of cases with rare CNVs preferentially affecting ASD/intellectual disability genes compared to $4.5 \%$ in controls (Fisher exact test $P=1.2 \times 10^{-3}$; Fig. $2 \mathrm{a}$ ). The observed enrichments did not change when potential case-control genome-wide differences for $\mathrm{CNV}$ rate and size were considered.

Our global analyses of these putative pathogenic loci use subjective boundaries for CNV overlap. Manual inspection of the data yields more accurate results. After eliminating CNVs that are less likely to have an aetiological role (heterozygous CNVs that disrupt autosomal recessive loci, events outside the critical region of overlap of genomic disorders, X-linked genes in females inherited from non-ASD fathers, duplications inherited from non-ASD parents, and intronic CNVs in NRXN1), 25 CNVs remained in the ASD group, compared to only four in the controls $\left(P=3.6 \times 10^{-6}\right.$; Supplementary Table 10$)$. Moreover, the latter four CNVs were all duplications at 1q21.1, 16p11.2 or $22 \mathrm{q} 11.2$, loci known to exhibit incomplete penetrance and variable expressivity ${ }^{6}$. The population attributable risk provided by the combination of all ASD CNVs that overlap ASDs and/or intellectual disability genes is estimated to be $3.3 \%$ (Supplementary Table 11). We also identified rare de novo chromosomal abnormalities and large CNVs likely to be aetiological (Supplementary Table 10).

We then tested for functional enrichment of gene sets among those genes affected by CNVs to identify biological processes involved in ASD (Fig. 3). Here, the term gene set refers to groups of genes that share a common function or operate in the same pathway. Such a functional enrichment mapping approach can combine single-gene effects into biologically meaningful groups ${ }^{21}$.

We compiled comprehensive collections of gene sets (Supplementary Table 12) and used the Fisher's exact test to assess which gene sets were more frequently affected by rare CNV events in ASD cases compared to controls. An estimate of the false-discovery rate (FDR) at each gene set was obtained by random permutation of case and control labels (Supplementary Information). To visualize enriched gene sets, overlap scores were used to organize these sets graphically into a functional enrichment map (or network) using Cytoscape ${ }^{22}$. We identified the 'seed' gene sets for the network at an FDR $q$-value of $5 \%$ and further relaxed the thresholds to $12.5 \%$ to better capture the network topology ${ }^{23}$.

Using these criteria only deletions were found to be significantly enriched in gene sets in cases over controls (Supplementary Fig. 5), consistent with the global burden results (Table 1). Specifically, 76 gene sets affected by deletions (2.18\% of sets tested) were found to be enriched and used to construct a functional map (Fig. 3a and Supplementary Figs 6 and 7). We tested for possible bias, including measures of CNV size and number for cases versus controls per gene set, as well as genome proximity, but no differences were found that

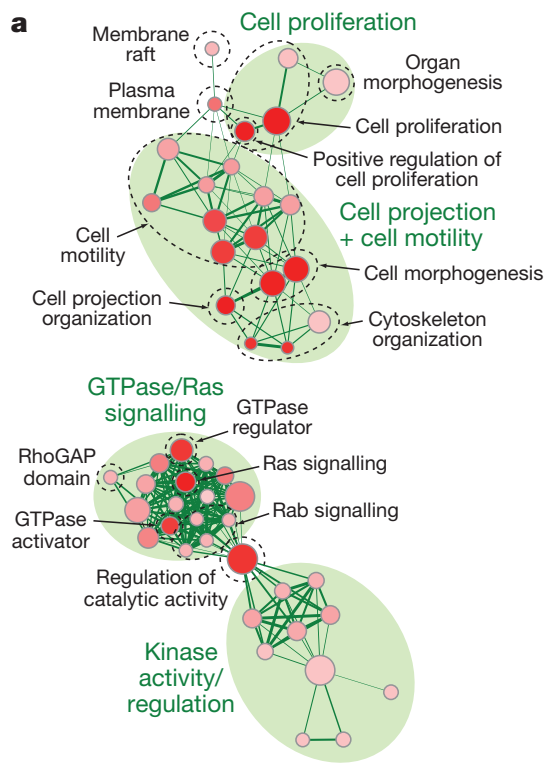

Figure 3 | A functional map of ASD. Enrichment results were mapped as a network of gene sets (nodes) related by mutual overlap (edges), where the colour (red, blue or yellow) indicates the class of gene set. Node size is proportional to the total number of genes in each set and edge thickness represents the number of overlapping genes between sets. a, Gene sets enriched for deletions are shown (red) with enrichment significance (FDR $q$-value) represented as a node colour gradient. Groups of functionally related gene sets are circled and labelled (groups, filled green circles; subgroups, dashed line). b, An expanded enrichment map shows the

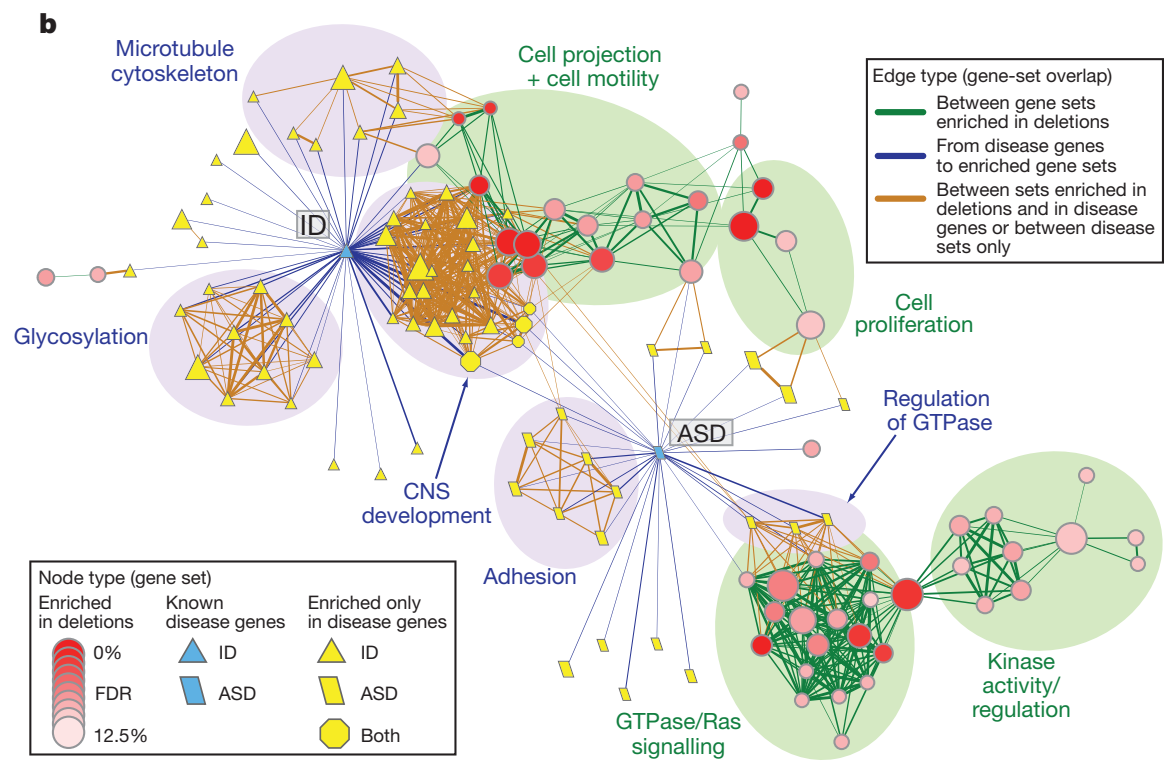

relationship between gene sets enriched in deletions (a) and sets of known ASD/intellectual disability genes. Node colour hue represents the class of gene set (that is, enriched in deletions, red; known disease genes (ASD and/or intellectual disability (ID) genes), blue; enriched only in disease genes, yellow). Edge colour represents the overlap between gene sets enriched in deletions (green), from disease genes to enriched sets (blue), and between sets enriched in deletions and in disease genes or between disease gene-sets only (orange). The major functional groups are highlighted by filled circles (enriched in deletions, green; enriched in ASD/intellectual disability, blue). 
might explain the observed enrichments (Supplementary Figs 8 and 9).

We identified enrichments in gene sets known to be involved in ASDs and also discovered new candidate ASD pathways (Fig. 3a and Supplementary Table 13). For example, gene sets involved in cell and neuronal development and function (including projection, motility and proliferation) previously reported in ASD-associated phenotypes were identified ${ }^{24}$. Novel observations included gene sets involved in GTPase/Ras signalling, with component Rho GTPases known to be involved in regulating dendrite and spine plasticity and associated with intellectual disability. We also found a tentative link to sets in the kinase activity/regulation functional group where only minorities of these sets meet a stringent 5\% FDR $q$-value threshold (Supplementary Fig. 10).

We further assessed the relationship of our functional enrichment map with known ASD/intellectual disability genes (Fig. $3 \mathrm{~b}$ and Supplementary Fig. 11) and found genes enriched in sets linked to microtubule cytoskeleton, glycosylation and CNS development/ adhesion $^{25}$. The two groups of genes found to be enriched in deletions (Fig. 3a) also displayed connectivity to the ASD/intellectual disability disease gene sets, either directly or through intermediates (Fig. 3b and Supplementary Fig. 12). Although ASD genes seem to be enriched in different subsets of genes compared to intellectual-disability-only genes, we cannot discount the possibility that this is the result of selection bias, and we expect that more intellectual disability genes may yet be linked to ASD.

Our findings provide strong support for the involvement of multiple rare genic CNVs, both genome-wide and at specific loci, in ASD. These findings, similar to those recently described in schizophrenia ${ }^{26}$, suggest that at least some of these ASD CNVs (and the genes that they affect) are under purifying selection ${ }^{27}$. Genes previously implicated in ASD by rare variant findings have pointed to functional themes in ASD pathophysiology ${ }^{6,28}$. Molecules such as NRXN1, NLGN3/4X and SHANK3, localized presynaptically or at the post-synaptic density (PSD), highlight maturation and function of glutamatergic synapses. Our data reveal that SHANK2, SYNGAP1 and DLGAP2 are new ASD loci that also encode proteins in the PSD. We also found intellectual disability genes to be important in $\mathrm{ASD}^{29}$. Furthermore, our functional enrichment map identifies new groups such as GTPase/Ras, effectively expanding both the number and connectivity of modules that may be involved in ASD. The next step will be to relate defects or patterns of alterations in these groups to ASD endophenotypes. The combined identification of higher-penetrance rare variants and new biological pathways, including those identified in this study, may broaden the targets amenable to genetic testing and therapeutic intervention.

\section{METHODS SUMMARY}

Raw data from ASD family (accession phs000267.v1.p1) and SAGE control (Accession: phs000092.v1.p1) genotyping are at NCBI dbGAP. CNVs were analysed using PLINK v1.07 $7^{30}$, R stats and custom scripts. See Supplementary Information for details. A list of all CNVs passing quality control is available in Supplementary Table 8.

\section{Received 3 December 2009; accepted 7 May 2010.}

Published online 9 June 2010.

1. Veenstra-Vanderweele, J., Christian, S. L. \& Cook, E. H. Jr. Autism as a paradigmatic complex genetic disorder. Annu. Rev. Genomics Hum. Genet. 5, 379-405 (2004)

2. Chakrabarti, S. \& Fombonne, E. Pervasive developmental disorders in preschool children: confirmation of high prevalence. Am. J. Psychiatry 162, 1133-1141 (2005).

3. Bailey, A. et al. Autism as a strongly genetic disorder: evidence from a British twin study. Psychol. Med. 25, 63-77 (1995).

4. Jamain, S. et al. Mutations of the X-linked genes encoding neuroligins NLGN3 and NLGN4 are associated with autism. Nature Genet. 34, 27-29 (2003).

5. Durand, C. M. et al. Mutations in the gene encoding the synaptic scaffolding protein SHANK3 are associated with autism spectrum disorders. Nature Genet. 39, 25-27 (2007).

6. Cook, E. H. Jr \& Scherer, S. W. Copy-number variations associated with neuropsychiatric conditions. Nature 455, 919-923 (2008).
7. Szatmari, P. et al. Mapping autism risk loci using genetic linkage and chromosomal rearrangements. Nature Genet. 39, 319-328 (2007).

8. Sebat, J. et al. Strong association of de novo copy number mutations with autism. Science 316, 445-449 (2007).

9. Marshall, C. R. et al. Structural variation of chromosomes in autism spectrum disorder. Am. J. Hum. Genet. 82, 477-488 (2008).

10. Weiss, L. A. et al. Association between microdeletion and microduplication at 16p11.2 and autism. N. Engl. J. Med. 358, 667-675 (2008).

11. Kumar, R. A. et al. Recurrent 16p11.2 microdeletions in autism. Hum. Mol. Genet. 17, 628-638 (2008)

12. Morrow, E. M. et al. Identifying autism loci and genes by tracing recent shared ancestry. Science 321, 218-223 (2008).

13. Wang, K. et al. Common genetic variants on 5 p14.1 associate with autism spectrum disorders. Nature 459, 528-533 (2009).

14. Weiss, L. A., Arking, D. E., Daly, M. J. \& Chakravarti, A. A genome-wide linkage and association scan reveals novel loci for autism. Nature 461, 802-808 (2009).

15. Bierut, L. J. et al. A genome-wide association study of alcohol dependence. Proc. Natl Acad. Sci. USA 107, 5082-5087 (2010).

16. Lee, A. B., Luca, D., Klei, L., Devlin, B. \& Roeder, K. Discovering genetic ancestry using spectral graph theory. Genet. Epidemiol. 34, 51-59 (2010).

17. Colella, S. et al. an Objective Bayes Hidden-Markov Model to detect and accurately map copy number variation using SNP genotyping data. Nucleic Acids Res. 35, 2013-2025 (2007)

18. Wang, K. et al. PennCNV: an integrated hidden Markov model designed for highresolution copy number variation detection in whole-genome SNP genotyping data. Genome Res. 17, 1665-1674 (2007).

19. Hamdan, F. F. et al. Mutations in SYNGAP1 in autosomal nonsyndromic mental retardation. N. Engl. J. Med. 360, 599-605 (2009).

20. Romorini, S. et al. A functional role of postsynaptic density-95-guanylate kinaseassociated protein complex in regulating Shank assembly and stability to synapses. J. Neurosci. 24, 9391-9404 (2004).

21. O'Dushlaine, C. et al. Molecular pathways involved in neuronal cell adhesion and membrane scaffolding contribute to schizophrenia and bipolar disorder susceptibility. Mol. Psychiatry doi:10.1038/mp.2010.7 (16 February 2010).

22. Cline, M. S. et al. Integration of biological networks and gene expression data using Cytoscape. Nature Protocols 2, 2366-2382 (2007).

23. Subramanian, A. et al. Gene set enrichment analysis: a knowledge-based approach for interpreting genome-wide expression profiles. Proc. Natl Acad. Sci. USA 102, 15545-15550 (2005)

24. Meechan, D. W., Tucker, E. S., Maynard, T. M. \& LaMantia, A. S. Diminished dosage of 22q11 genes disrupts neurogenesis and cortical development in a mouse model of 22q11 deletion/DiGeorge syndrome. Proc. Natl Acad. Sci. USA 106, 16434-16445 (2009)

25. Wegiel, J. et al. The neuropathology of autism: defects of neurogenesis and neuronal migration, and dysplastic changes. Acta Neuropathol. doi:10.1007/ s00401-010-0655-4 (3 March 2010)

26. International Schizophrenia Consortium. Rare chromosomal deletions and duplications increase risk of schizophrenia. Nature 455, 237-241 (2008).

27. Conrad, D. F. et al. Origins and functional impact of copy number variation in the human genome. Nature 464, 704-712 (2010).

28. Glessner, J. T. et al. Autism genome-wide copy number variation reveals ubiquitin and neuronal genes. Nature 459, 569-573 (2009).

29. Skuse, D. H. Rethinking the nature of genetic vulnerability to autistic spectrum disorders. Trends Genet. 23, 387-395 (2007).

30. Purcell, S. et al. PLINK: a tool set for whole-genome association and populationbased linkage analyses. Am. J. Hum. Genet. 81, 559-575 (2007).

Supplementary Information is linked to the online version of the paper at www.nature.com/nature.

Acknowledgements The authors acknowledge the families participating in the study and the main funders of the Autism Genome Project Consortium (AGP) Autism Speaks (USA), the Health Research Board (HRB; Ireland), The Medical Research Council (MRC; UK), Genome Canada/Ontario Genomics Institute, and the Hilibrand Foundation (USA). Additional support for individual groups was provided by the US National Institutes of Health (NIH grants HD055751, HD055782, HD055784, HD35465, MH52708, MH55284, MH57881, MH061009, MH06359, MH066673, MH080647, MH081754, MH66766, NS026630, NS042165, NS049261), the Canadian Institute for Advanced Research (CIFAR), the Canadian Institutes for Health Research (CIHR), Assistance Publique-Hôpitaux de Paris (France), Autistica, Canada Foundation for Innovation/Ontario Innovation Trust, Deutsche Forschungsgemeinschaft (grant Po 255/17-4) (Germany), EC Sixth FP AUTISM MOLGEN, Fundação Calouste Gulbenkian (Portugal), Fondation de France, Fondation FondaMental (France) Fondation Orange (France), Fondation pour la Recherche Médicale (France), Fundação para a Ciência e Tecnologia (Portugal), the Hospital for Sick Children Foundation and University of Toronto (Canada), INSERM (France), Institut Pasteur (France), the Italian Ministry of Health (convention 181 of 19.10.2001), the John P Hussman Foundation (USA), McLaughlin Centre (Canada), Ontario Ministry of Research and Innovation (Canada), the Seaver Foundation (USA), the Swedish Science Council, The Centre for Applied Genomics (Canada), the Utah Autism Foundation (USA) and the Wellcome Trust core award 075491/Z/04 (UK). D.P. is supported by fellowships from the Royal Netherlands Academy of Arts and 
Sciences (TMF/DA/5801) and the Netherlands Organization for Scientific Research (Rubicon 825.06.031). S.W.S. holds the GlaxoSmithKline-CIHR Pathfinder Chair in Genetics and Genomics at the University of Toronto and the Hospital for Sick Children (Canada).

Author Contributions D.P., J.D.B., R.M.C., E.H.C., H.C., M.C., B.D., S.E., L.G., D.H.G., M.G., J.L.H., J.H., J.M., A.P.M., J.I.N., A.D.P., M.A.P.-V., G.D.S., P.S., A.M.V., V.J.V., E.M.W., J.S.S., C.B. and S.W.S. were leading contributors in the design, analysis and writing of this study. A.J.B., A.B., G.D., C.M.F., H.H., S.M.K., E.M., S.F.N., G.O., J.P., T.H.W., J.D.B., R.M.C., E.H.C., H.C., B.D., S.E., L.G., D.H.G., M.G., J.L.H., J.H., A.P.M., J.I.N., A.D.P., M.A.P.-V., G.D.S., P.S., A.M.V., V.J.V., E.M.W., S.W.S., J.S.S. and C.B. are Lead Autism Genome Project Consortium (AGP) investigators who contributed equally to this project. All other authors were either involved in phenotype and clinical assessments or have participated in experiments and analysis.

Author Information Reprints and permissions information is available at www.nature.com/reprints. The authors declare competing financial interests: details accompany the full-text HTML version of the paper at www.nature.com/ nature. Readers are welcome to comment on the online version of this article at www.nature.com/nature. Correspondence and requests for materials should be addressed to S.W.S. (stephen.scherer@sickkids.ca)

Dalila Pinto ${ }^{1}$, Alistair T. Pagnamenta ${ }^{2}$, Lambertus Klei ${ }^{3}$, Richard Anney ${ }^{4}$, Daniele Merico $^{5}$, Regina Regan ${ }^{6}$, Judith Conroy ${ }^{6}$, Tiago R. Magalhaes ${ }^{7,8}$, Catarina Correia ${ }^{7,8}$, Brett S. Abrahams ${ }^{9}$, Joana Almeida ${ }^{10}$, Elena Bacchelli" , Gary D. Bader ${ }^{5,72}$, Anthony J. Bailey $^{12}$, Gillian Baird ${ }^{13}$, Agatino Battaglia ${ }^{14}$, Tom Berney ${ }^{15,56}$, Nadia Bolshakova ${ }^{4}$, Sven Bölte $^{16}$, Patrick F. Bolton ${ }^{17}$, Thomas Bourgeron ${ }^{18}$, Sean Brennan ${ }^{4}$, Jessica Brian ${ }^{19}$, Susan E. Bryson ${ }^{20}$, Andrew R. Carson', Guillermo Casallo', Jillian Casey ${ }^{6}$, Brian H.Y. Chung ${ }^{1}$ Lynne Cochrane ${ }^{4}$, Christina Corsello ${ }^{21}$, Emily L. Crawford ${ }^{22}$, Andrew Crossett ${ }^{23}$, Cheryl Cytrynbaum', Geraldine Dawson ${ }^{24,25}$, Maretha de Jonge ${ }^{26}$, Richard Delorme ${ }^{27}$, Irene Drmic $^{19}$, Eftichia Duketis $^{16}$, Frederico Duque ${ }^{10}$, Annette Estes $^{28}$, Penny Farrar ${ }^{2}$, Bridget $^{\prime}$ A. Fernandez ${ }^{29}$, Susan E. Folstein ${ }^{30}$, Eric Fombonne ${ }^{31}$, Christine M. Freitag ${ }^{16}$, John Gilbert $^{30}$, Christopher Gillberg ${ }^{32}$, Joseph T. Glessner ${ }^{33}$, Jeremy Goldberg ${ }^{34}$,'Andrew Green $^{6}$, Jonathan Green ${ }^{35}$, Stephen J. Guter ${ }^{36}$, Hakon Hakonarson ${ }^{33,37}$, Elizabeth A Heron $^{4}$, Matthew Hill ${ }^{4}$, Richard Holt ${ }^{2}$, Jennifer L. Howe', Gillian Hughes ${ }^{4}$, Vanessa Hus $^{21}$, Roberta Igliozzi ${ }^{14}$, Cecilia Kim ${ }^{33}$, Sabine M. Klauck ${ }^{38}$, Alexander Kolevzon ${ }^{39}$, Olena Korvatska ${ }^{40}$, Vlad Kustanovich ${ }^{41}$, Clara M. Lajonchere ${ }^{41}$, Janine A. Lamb ${ }^{42}$, Magdalena Laskawiec ${ }^{12}$, Marion Leboyer ${ }^{43}$, Ann Le Couteur ${ }^{15,56}$, Bennett L. Leventhal ${ }^{44,45}$, Anath C. Lionel ${ }^{1}$, Xiao-Qing Liu' ${ }^{1}$, Catherine Lord ${ }^{21}$, Linda Lotspeich ${ }^{46}$ Sabata C. Lund ${ }^{22}$, Elena Maestrini ${ }^{11}$, William Mahoney ${ }^{47}$, Carine Mantoulan ${ }^{48}$, Christian R. Marshall1, Helen McConachie ${ }^{15,56}$, Christopher J. McDougle ${ }^{49}$, Jane McGrath ${ }^{4}$, William M. McMahon ${ }^{50}$, Alison Merikangas ${ }^{4}$, Ohsuke Migita', Nancy J. Minshew $^{51}$, Ghazala K. Mirza ${ }^{2}$, Jeff Munson ${ }^{52}$, Stanley F. Nelson ${ }^{53}$, Carolyn Noakes ${ }^{19}$, Abdul Noor ${ }^{54}$, Gudrun Nygren ${ }^{32}$, Guiomar Oliveira ${ }^{10}$, Katerina Papanikolaou ${ }^{55}$, Jeremy R. Parr ${ }^{56}$, Barbara Parrini ${ }^{14}$, Tara Paton ${ }^{1}$, Andrew Pickles ${ }^{57}$, Marion Pilorge ${ }^{58}$, Joseph Piven $^{59}$, Chris P. Ponting ${ }^{60}$, David J. Posey ${ }^{49}$, Annemarie Poustka ${ }^{38}+$, Fritz Poustka ${ }^{16}$, Aparna Prasad ${ }^{1}$, Jiannis Ragoussis ${ }^{2}$, Katy Renshaw ${ }^{12}$, Jessica Rickaby ${ }^{1}$, Wendy Roberts $^{19}$, Kathryn Roeder ${ }^{23}$, Bernadette Roge ${ }^{48}$, Michael L. Rutter $^{61}$, Laura J. Bierut ${ }^{62}$, John P. Rice ${ }^{62}$, Jeff Salt ${ }^{36}$, Katherine Sansom', Daisuke Sato ${ }^{1}$, Ricardo Segurado ${ }^{4}$, Ana F Sequeira ${ }^{7,8}$, Lili Senman ${ }^{19}$, Naisha Shah ${ }^{6}$, Val C. Sheffield ${ }^{63}$, Latha Soorya ${ }^{39}$, Inês Sousa ${ }^{2}$, Olaf Stein ${ }^{64}$, Nuala Sykes ${ }^{2}$, Vera Stoppioni ${ }^{65}$, Christina Strawbridge ${ }^{34}$, Raffaella Tancredi ${ }^{14}$, Katherine Tansey ${ }^{4}$, Bhooma Thiruvahindrapduram', Ann P. Thompson ${ }^{34}$, Susanne Thomson ${ }^{22}$, Ana Tryfon ${ }^{39}$, John Tsiantis ${ }^{55}$, Herman Van Engeland ${ }^{26}$, John B. Vincent ${ }^{54}$, Fred Volkmar ${ }^{66}$, Simon Wallace ${ }^{12}$, Kai Wang ${ }^{33}$, Zhouzhi Wang ${ }^{1}$, Thomas H. Wassink ${ }^{67}$, Caleb Webber ${ }^{60}$, Rosanna Weksberg ${ }^{1}$, Kirsty Wing ${ }^{2}$ Kerstin Wittemeyer ${ }^{48}$, Shawn Wood ${ }^{3}$, Jing Wu ${ }^{23}$, Brian L. Yaspan ${ }^{22}$, Danielle Zurawiecki ${ }^{39}$, Lonnie Z'waigenbaum ${ }^{68}$, Joseph D. Buxbaum ${ }^{39}$, Rita M. Cantor ${ }^{53}$, Edwin H. Cook ${ }^{36}$, Hilary Coon ${ }^{50}$, Michael L. Cuccaro ${ }^{30}$, Bernie Devlin ${ }^{3}$, Sean Ennis ${ }^{6}$, Louise Gallagher $^{4}$, Daniel H. Geschwind ${ }^{9}$, Michael Gill ${ }^{4}$, Jonathan L. Haines ${ }^{69}$, Joachim Hallmayer ${ }^{46}$, Judith Miller ${ }^{50}$, Anthony P. Monaco ${ }^{2}$, John I. Nurnberger Jr ${ }^{49}$, Andrew D. Paterson', Margaret A. Pericak-Vance ${ }^{30}$, Gerard D. Schellenberg ${ }^{70}$, Peter Szatmari ${ }^{34}$ Astrid M. Vicente ${ }^{7,8}$, Veronica J. Vieland ${ }^{64}$, Ellen M. Wijsman ${ }^{71}$, Stephen W. Scherer ${ }^{1,72}$, James S. Sutcliffe ${ }^{22} \&$ Catalina Betancur ${ }^{58}$

The Centre for Applied Genomics and Program in Genetics and Genomic Biology, The Hospital for Sick Children, Toronto, Ontario M5G 1L7, Canada. ${ }^{2}$ Wellcome Trust Centre for Human Genetics, University of Oxford, Oxford OX3 7BN, UK. ${ }^{3}$ Department of Psychiatry, University of Pittsburgh School of Medicine, Pittsburgh, Pennsylvania 15213, USA. ${ }^{4}$ Autism Genetics Group, Department of Psychiatry, School of Medicine, Trinity College, Dublin 8, Ireland. ${ }^{5}$ Banting and Best Department of Medical Research, Terrence Donnelly Centre for Cellular and Biomolecular Research, University of Toronto, Toronto, Ontario M5S 3E1, Canada. ${ }^{6}$ School of Medicine and Medical Science University College, Dublin 4, Ireland. ${ }^{7}$ Instituto Nacional de Saude Dr Ricardo Jorge 1649-016 Lisbon and Instituto Gulbenkian de Cîencia, 2780-156 Oeiras, Portugal. ${ }^{8}$ BioFIG-Center for Biodiversity, Functional and Integrative Genomics, Campus da FCUL, C2.2.12, Campo Grande, 1749-016 Lisboa, Portugal. 'P Program in Neurogenetics, Department of Neurology and Center for Autism Research and Treatment, Semel Institute, David Geffen School of Medicine at UCLA, Los Angeles, California 90095, USA. ${ }^{10}$ Hospital Pediátrico de Coimbra, 3000 - 076 Coimbra, Portugal. ${ }^{11}$ Department of Biology, University of Bologna, 40126 Bologna, Italy. ${ }^{12}$ Department of Psychiatry, University of Oxford, Warneford Hospital, Headington, Oxford OX3 7JX, UK. ${ }^{13}$ Newcomen Centre, Guy's
Hospital, London SE1 9RT, UK. ${ }^{14}$ Stella Maris Institute for Child and Adolescent Neuropsychiatry, 56128 Calambrone (Pisa), Italy. ${ }^{15} \mathrm{Child}$ and Adolescent Mental Health University of Newcastle, Sir James Spence Institute, Newcastle upon Tyne NE1 4LP, UK ${ }^{16}$ Department of Child and Adolescent Psychiatry, Psychosomatics and Psychotherapy, J.W. Goethe University Frankfurt, 60528 Frankfurt, Germany. ${ }^{17}$ Department of Child and Adolescent Psychiatry, Institute of Psychiatry, London SE5 8AF, UK. ${ }^{18}$ Human Genetics and Cognitive Functions, Institut Pasteur; University Paris Diderot-Paris 7, CNRS URA 2182, Fondation FondaMental, 75015 Paris, France. ${ }^{19}$ Autism Research Unit, The Hospital for Sick Children and Bloorview Kids Rehab, University of Toronto, Toronto, Ontario M5G $1 \mathrm{X} 8$, Canada. ${ }^{20}$ Department of Pediatrics and Psychology, Dalhousie University, Halifax, Nova Scotia B3K 6R8, Canada. ${ }^{21}$ Autism and Communicative Disorders Centre, University of Michigan, Ann Arbor, Michigan 48109-2054, USA. ${ }^{22}$ Department of Molecular Physiology and Biophysics, Vanderbilt Kennedy Center, and Centers for Human Genetics Research and Molecular Neuroscience, Vanderbilt University, Nashville, Tennessee 37232, USA. ${ }^{23}$ Department of Statistics, Carnegie Mellon University, Pittsburgh, Pennsylvania 15213, USA. ${ }^{24}$ Autism Speaks, New York 10016, USA. ${ }^{25}$ Department of Psychiatry, University of North Carolina, Chapel Hill, North Carolina 27599-3366, USA. ${ }^{26}$ Department of Child Psychiatry, University Medical Center, Utrecht 3508 GA, The Netherlands. ${ }^{27}$ INSERM U 955, Fondation FondaMental, APHP, Hôpital Robert Debré, Child and Adolescent Psychiatry, 75019 Paris, France. ${ }^{28}$ Department of Speech and Hearing Sciences, University of Washington, Seattle, Washington 98195, USA. ${ }^{29}$ Disciplines of Genetics and Medicine, Memorial University of Newfoundland, St John's Newfoundland A1B 3V6, Canada. ${ }^{30}$ The John P. Hussman Institute for Human Genomics, University of Miami, Miami, Florida 33101, USA. ${ }^{31}$ Division of Psychiatry, McGill University, Montreal, Quebec H3A 1A1, Canada. ${ }^{32}$ Department of Child and Adolescent Psychiatry, Göteborg University, Göteborg S41345, Sweden. ${ }^{33}$ The Center for Applied Genomics, Division of Human Genetics, The Children's Hospital of Philadelphia, Philadelphia, Pennsylvania 19104, USA.

${ }^{34}$ Department of Psychiatry and Behavioural Neurosciences, McMaster University, Hamilton, Ontario L8N 3Z5, Canada. ${ }^{35}$ Academic Department of Child Psychiatry, Booth Hall of Children's Hospital, Blackley, Manchester M9 7AA, UK. ${ }^{36}$ Institute for Juvenile Research, Department of Psychiatry, University of Illinois at Chicago, Chicago, Illinois 60612, USA. ${ }^{37}$ Department of Pediatrics, Children's Hospital of Philadelphia, University of Pennsylvania School of Medicine, Philadelphia, Pennsylvania 19104, USA. ${ }^{38}$ Division of Molecular Genome Analysis, German Cancer Research Center (DKFZ), Heidelberg 69120, Germany. ${ }^{39}$ The Seaver Autism Center for Research and Treatment, Department of Psychiatry, Mount Sinai School of Medicine, New York 10029, USA. ${ }^{40}$ Department of Medicine, University of Washington, Seattle, Washington 98195, USA. ${ }^{41}$ Autism Genetic Resource Exchange, Autism Speaks, Los Angeles, California 90036-4234, USA. ${ }^{42}$ Centre for Integrated Genomic Medical Research, University of Manchester, Manchester M13 9PT, UK. ${ }^{43}$ INSERM U995, Department of Psychiatry, Groupe Hospitalier Henri Mondor-Albert Chenevier, AP-HP; University Paris 12, Fondation FondaMental, Créteil 94000, France. ${ }^{44}$ Nathan Kline Institute for Psychiatric Research (NKI), 140 Old Orangeburg Road, Orangeburg, New York 10962, USA. ${ }^{45}$ Department of Child and Adolescent Psychiatry, New York University and NYU Child Study Center, 550 First Avenue, New York, New York 10016, USA. ${ }^{46}$ Department of Psychiatry, Division of Child and Adolescent Psychiatry and Child Development, Stanford University School of Medicine, Stanford, California 94304, USA. ${ }^{47}$ Department of Pediatrics, McMaster University, Hamilton, Ontario L8N 3Z5, Canada. ${ }^{48}$ Centre d'Eudes et de Recherches en Psychopathologie, University de Toulouse Le Mirail, Toulouse 31200, France.

${ }^{49}$ Department of Psychiatry, Indiana University School of Medicine, Indianapolis, Indiana 46202, USA. ${ }^{50}$ Psychiatry Department, University of Utah Medical School, Salt Lake City, Utah 84108, USA. ${ }^{51}$ Departments of Psychiatry and Neurology, University of Pittsburgh School of Medicine, Pittsburgh, Pennsylvania 15213, USA. ${ }^{52}$ Department of Psychiatry and Behavioural Sciences, University of Washington, Seattle, Washington 98195, USA. ${ }^{53}$ Department of Human Genetics, University of California-Los Angeles School of Medicine, Los Angeles, California 90095, USA. ${ }^{54}$ Centre for Addiction and Mental Health, Clarke Institute and Department of Psychiatry, University of Toronto, Toronto, Ontario M5G 1X8, Canada. ${ }^{55}$ University Department of Child Psychiatry, Athens University, Medical School, Agia Sophia Children's Hospital, 11527 Athens, Greece. ${ }^{56}$ Insitutes of Neuroscience and Health and Society, Newcastle University, Newcastle Upon Tyne NE1 7RU, UK. ${ }^{57}$ Department of Medicine, School of Epidemiology and Health Science, University of Manchester, Manchester M13 9PT, UK. ${ }^{58}$ INSERM U952 and CNRS UMR 7224 and UPMC Univ Paris 06, Paris 75005, France. ${ }^{59}$ Carolina Institute for Developmental Disabilities, University of North Carolina at Chapel Hill, North Carolina 27599-3366, USA. ${ }^{60}$ MRC Functional Genomics Unit, Department of Physiology, Anatomy and Genetics, University of Oxford, Oxford OX13QX, UK. ${ }^{61}$ Social, Genetic and Developmental Psychiatry Centre, Institute Of Psychiatry, London SE5 8AF, UK. ${ }^{62}$ Department of Psychiatry, Washington University in St Louis, School of Medicine, St Louis, Missouri 63130, USA. ${ }^{63}$ Department of Pediatrics and Howard Hughes Medical Institute Carver College of Medicine, University of lowa, lowa City, lowa 52242, USA.

${ }^{64}$ Battelle Center for Mathematical Medicine, The Research Institute at Nationwide Children's Hospital and The Ohio State University, Columbus, Ohio 43205, USA. ${ }^{65}$ Neuropsichiatria Infantile, Ospedale Santa Croce, 61032 Fano, Italy. ${ }^{66}$ Child Study Centre, Yale University, New Haven, Connecticut 06520, USA. ${ }^{67}$ Department of Psychiatry, Carver College of Medicine, lowa City, lowa 52242, USA. ${ }^{68}$ Department of Pediatrics, University of Alberta, Edmonton, Alberta T6G 2J3, Canada. ${ }^{69}$ Center for Human Genetics Research, Vanderbilt University Medical Centre, Nashville, Tennessee 37232, USA. ${ }^{70}$ Pathology and Laboratory Medicine, University of Pennsylvania, Pennsylvania 19104, USA. ${ }^{71}$ Departments of Biostatistics and Medicine, University of Washington, Seattle, Washington 98195, USA. ${ }^{72}$ Department of Molecular Genetics, University of Toronto, Toronto, Ontario M5S 1A1, Canada. tDeceased. 